\title{
VARIAÇÃO ESPAÇO-TEMPORAL DE POPULAÇÕES DE HYPNEA MUSCIFORMIS (RHODOPHYTA, GIGARTINALES) NA BAÍA DE SEPETIBA E ARMAÇÃO DOS BÚZIOS, RJ, BRASIL ${ }^{1}$
}

\author{
Renata Perpetuo Reis ${ }^{2}$ \\ Yocie Yoneshigue-Valentin ${ }^{3}$
}

Recebido em 03/03/1999. Aceito em 01/07/1999

RESUMO - (Variação espaço-temporal de populações de Hypnea musciformis (Rhodophyta, Gigartinales) na baía de Sepetiba e Armação dos Búzios, RJ, Brasil). Três populaçōes de Hypnea musciformis foram analisadas ao longo do Estado do Rio de Janeiro; duas epifíticas, sublitorâneas, localizadas na baía de Sepetiba (município de Mangaratiba) e uma epilítica, litorânea, localizada em praia exposta a mar aberto (praia Rasa, município de Armação de Búzios). Essas populações foram analisadas quanto à produção de biomassa, flora acompanhante e as possíveis interaçōes com alguns fatores ambientais. As biomassas de $H$. musciformis variaram nos três locais, não havendo padrão sazonal comum. Observou-se que a movimentação de água, insolação, flora acompanhante e a relação entre a epífita e o hospedeiro, Sargassum spp., causaram efeitos diferenciados sobre a produção das biomassas nas diferentes populaçōes.

Palavras chave - Hypnea musciformis, carragenófita, sazonalidade, biomassa

ABSTRACT - (Spatial and temporal variation of populations of Hypnea musciformis (Rhodophyta, Gigartinales) in the Sepetiba bay and "Armação dos Búzios", RJ, Brazil). Three populations of $H$. musciformis were analyzed throughout Rio de Janeiro State; two sublittoral epiphytic populations located in the Sepetiba bay (Mangaratiba municipality), and one intertidal epilithic population on the shore facing the open sea (Rasa beach, Armação dos Búzios municipality). These populations were analyzed for seasonality in biomass production, the accompanying flora species, and the possible interactions with some environmental factors. The biomass production of $H$. musciformis was different at each site, water movement, sunlight, accompanying flora, and the relation between the epiphyte and host, Sargassum spp., caused different effects on biomass production in different populations.

Key words - Hypnea musciformis, carragenophyte, seasonality, biomass

\section{Introdução}

O litoral brasileiro estende-se por cerca de $8.000 \mathrm{~km}$, caracterizado por diversos tipos ambientais e grande diversidade específica, na qual são incluídas várias espécies

1 Parte de Tese de Doutorado da primeira Autora

2 Instituto de Pesquisas Jardim Botânico do Rio de Janeiro/Ministério do Meio Ambiente, Rua Pacheco Leão, 915, CEP 22.460-030, Rio de Janeiro, RJ, Brasil

3 Departamento de Botânica, IB, Universidade Federal do Rio de Janeiro, CEP 21941-900. Rio de Janeiro, RJ, Brasil 
de valor econômico, justificando, assim a necessidade de estudar o potencial de seus recursos, visando o seu conhecimento e manejo. Dentro deste contexto, evidencia-se a potencialidade de $H$. musciformis em bancos naturais no litoral do Estado do Rio de Janeiro, como fonte alternativa para obtenção de matéria-prima para a comercialização de carragenana (Oliveira Filho et al. 1992).

Para conhecer a interferência de algumas das condições ambientais no desenvolvimento desta alga e, conseqüentemente, subsidiar a sua exploração racional, têm sido realizados estudos sobre a variação temporal da biomassa de $H$. musciformis em populações naturais na Índia (Rao 1970), Havaí (Mshigeni 1977), EUA (Durako \& Dawes 1980) e Brasil, nos Estados de São Paulo (Schenkman 1980; 1989; Faccini 1998) e da Bahia (Wallner et al. 1992).

Essa produção foi, também, analisada em cultivos extensivos no mar, no Caribe (Humm \& Kreuzer 1975), Israel (Friedlander \& Lipkin 1982; Friedlander \& Zelikovith 1984), EUA (Guist et al. 1982) e Brasil, nos Estados de São Paulo (Oliveira \& Berchez 1987; Berchez 1990; Berchez \& Oliveira 1990; Berchez et al. 1993), do Rio Grande do Norte (Câmara Neto et al. 1980; Lima et al. 1981; Câmara Neto 1987) e da Bahia (Wallner et al. 1992).

Tem sido estudada, também, a variação temporal da biomassa, através de cultivos em pequena escala, como em tanques, nas ilhas Virgens (Haines 1975; Langston et al. 1977), Israel (Guist et al. 1982) e Brasil, no Estado de São Paulo (Oliveira et al. 1990), além de cultivos em condições controladas de laboratório (Schenkman 1986; Yokoya 1989).

No litoral norte do Estado de São Paulo, uma população foi manejada sendo avaliado seu estoque, a sua recuperação e observada a distribuição espacial da espécie (Faccini 1998). Contudo, torna-se imprescindível estudos complementares sobre outras populações da costa brasileira, visando conhecer outros aspectos da biologia de $H$. musciformis, identificar o potencial desta espécie ao longo do litoral brasileiro, de modo a subsidiar ações de manejo.

Nesse sentido, o objetivo deste estudo foi analisar a variação da biomassa de $H$. musciformis e a sua flora acompanhante nas quatro estações do ano em locais que apresentam diferentes regimes de movimentação de água e exposição ao ar, em trechos do Estado do Rio de Janeiro.

\section{Material e métodos}

Foram analisadas três populações de H. musciformis no Estado do Rio de Janeiro, Brasil (Fig. 1). Duas na baía de Sepetiba, no município de Mangaratiba, na praia Grande e praia Vermelha, (Fig. 1D) e outra no município de Armação dos Búzios, na praia Rasa (Fig. 1C). Comparando as praias, a praia Rasa caracterizou-se por estar situada em costão rochoso exposto a mar aberto e por apresentar espécies de algas características de locais com alta movimentação de água, como Pterocladiella capillacea (S. G. Gmel.) Santel. \& Hommers., Levringia brasiliensis (Montagne) A. B. Joly e Chaetomorpha antennina (Bory) Kützing (Oliveira 1977; Ugadim 1985).

A praia Rasa situa-se no município de Armação de Búzios (Fig. 1C, estação de coleta 1), está voltada para sudoeste e possui leve inclinação de substrato, 


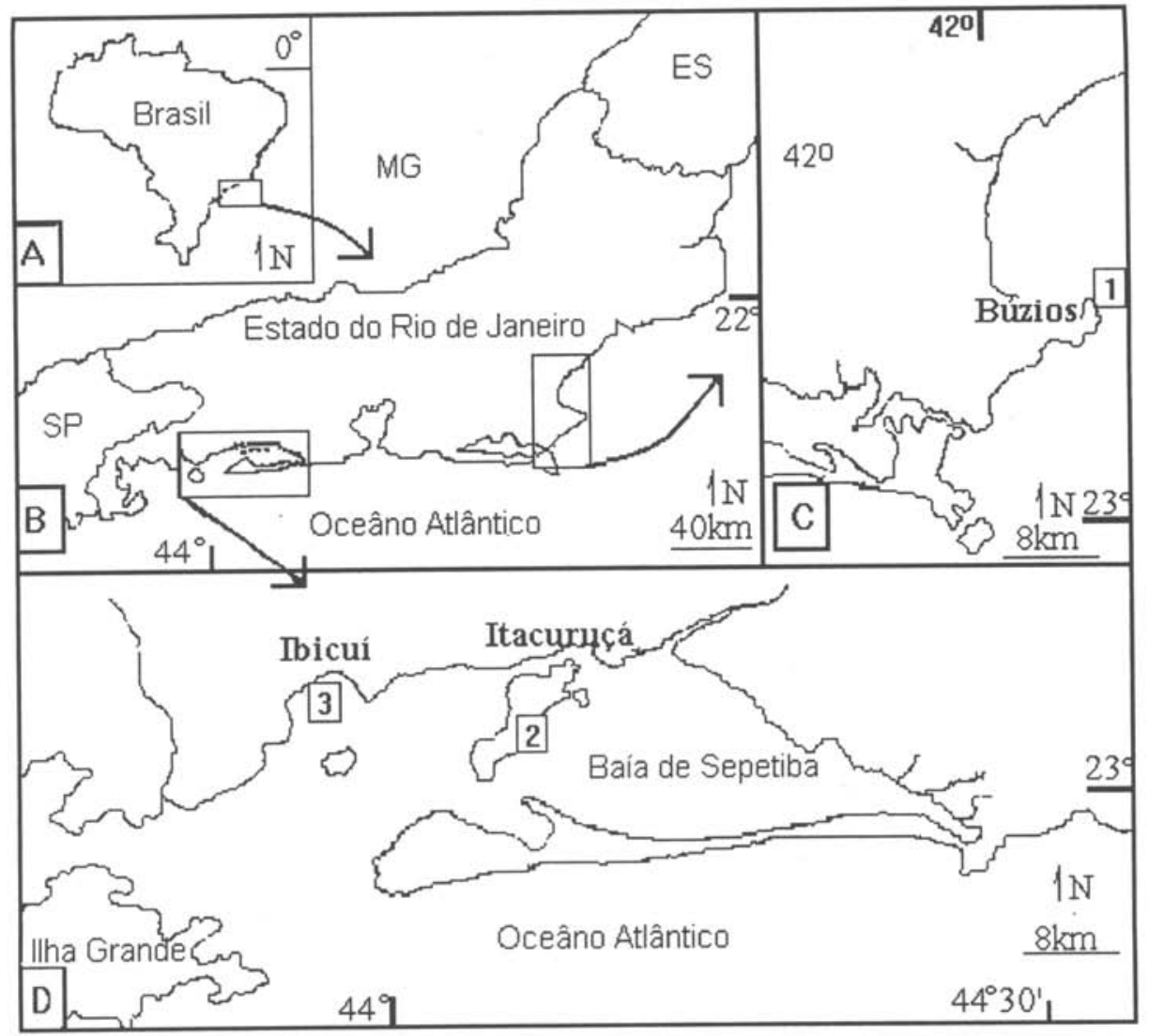

Figura 1. Localização das estações de coleta: (A) Brasil; (B) Estado do Rio de Janeiro; (C) detalhe do município de Armação dos Búzios com a estação de coleta $(1)=$ praia Rasa; (D) detalhe do município de Mangaratiba com as estações de coletas (2) = praia Grande, Ilha de Itacuruçá, na cidade de Itacuruçá e (3) $=$ praia Vermelha, na cidade de Ibicuí.

aproximadamente $15^{\circ}$ de inclinação. A amplitude vertical das marés varia de 0,50 a $0,90 \mathrm{~m}$. Em razão da pouca profundidade, a fraca agitação superficial de água provoca turbidez quase constante na água (Yoneshigue 1985). A população estudada de $H$. musciformis ocorre como epilítica, formando densos tapetes, emaranhada com outras algas, na região litorânea, ficando exposta ao ar em até $30 \mathrm{~cm}$ acima do nível das baixamares de sizígia.

A baía de Sepetiba, onde estão incluídas as outras duas estações de coleta possui $447 \mathrm{~km}^{2}$ de área, tendo como características hidrodinâmicas um padrão de circulação horário, influenciado pelas marés e entrada de águas fluviais na região central da baía. Nesta baía, as flutuações sazonais de aporte de água doce são atenuadas (Barcellos 1995). A praia Grande localiza-se na ilha de Itacuruçá, situada ao norte da baía (Fig. 1D, estação de coleta 2), na região sudoeste da ilha. As coletas foram executadas no costão leste desta praia, que se caracteriza por ser local abrigado a maior parte do ano, sendo escolhido 
para ancoradouro em épocas de mar agitado. A água é clara, tornando-se mais turva com a subida da maré, que revolve o fundo. O substrato é formado por vários matacões com ângulo de declividade de $15 \mathrm{a} 30^{\circ}$. Nesses matacões existem extensos bancos de Sargassum spp., que são os principais hospedeiros de $H$. musciformis, formando faixa de aproximadamente $3 \mathrm{~m}$ larg., estendendo-se por quase todo o costão desta praia.

A praia Vermelha situa-se nas proximidades da ilha Guaíba, próxima à entrada da baía de Sepetiba (Fig. 1D, estação de coleta 3). O substrato desta praia é formado, em geral, por vários matacões irregulares, com largura superior a $1 \mathrm{~m}$. O conjunto de matacões tende à inclinação em torno de $30^{\circ}$. As populações epifíticas de $H$. musciformis têm como seu principal hospedeiro Sargassum spp., formando bancos descontínuos junto a outras algas, com largura aproximada de $3 \mathrm{~m}$. A diferença entre a movimentação de água das praias da baía de Sepetiba foi obtida através do experimento com esferas de gesso, seguindo a metodologia empregada por Berchez (1990). Neste experimento, a praia Grande apresentou a menor movimentação de água.

As coletas foram bimestrais e realizadas entre agosto/1994 e agosto/1996 na praia Rasa e nas praias da baía de Sepetiba no período de junho/1994 a junho/ 1996. Na praia Rasa as coletas foram realizadas durante as baixamares, devido ao tipo de ondulações no costão muito inclinado e a forte turbidez da água que impossibilitam trabalhar neste local com maré alta. Nas praias da baía de Sepetiba, as coletas foram realizadas através de mergulho autônomo.

Adotou-se a metodologia de amostragem destrutiva de algas por meio de quadrados aleatórios (De Wreede 1985; Tsuda \& Abbot 1985), método normalmente utilizado em estudos de populações monoespecíficas com talo de pequeno porte (Chapman 1985; De Wreede 1985). Utilizou-se sete quadrados com $0,25 \mathrm{~m}^{2}$, dispostos aleatoriamente em um transecto de linha horizontal, com $20 \mathrm{~m}$, colocado no meio da faixa da população de $H$. musciformis. O cálculo do número de amostras necessárias para a estimativa da média da população de $H$. musciformis foi obtido através da representação gráfica da média acumulada da biomassa, em massa seca de $H$. musciformis, em relação ao número acumulado de amostras (De Wreede 1985; Chapman 1985). A dinâmica sazonal de $H$. musciformis foi quantificada através da biomassa da população seca em estufa até a obtenção de massa constante.

As macroalgas que ocorrem sobre o substrato rochoso junto a $\mathrm{H}$. musciformis foram identificadas, de modo a estabelecer o efeito na variação da biomassa de $H$. musciformis e das macroalgas acompanhantes, nas diferentes épocas do ano e nos diferentes locais de coleta, através do percentual de massa seca das algas nos quadrados. A identificação dessas macroalgas seguiu as categorias taxonômicas adotadas por Wynne (1998). Neste estudo, as espécies com poucos centímetros de altura e de aparência filamentosa foram agrupadas e denominadas "filamentosa" (Tab. 1). As coralináceas articuladas não foram consideradas, uma vez que a sua biomassa não foi expressiva nos locais amostrados. As espécies acompanhantes mais representativas foram relacionadas na Tab. 2.

Os dados meteorológicos utilizados foram fornecidos pelo Instituto Nacional de Meteorologia, Distrito Sexto de Meteorologia, do Ministério da Agricultura e do Abastecimento (INMET) e pelo Banco de Dados Oceanográficos da Diretoria de Hidrografia e Navegação, do Ministério da Marinha (DHN), conforme ilustrados na Fig. 2. 
Tabela 1. Lista de táxons de algas da flora acompanhante de $H$. musciformis nas três estações de coleta, agrupados em filamentosa, representados nas figuras 3 a 7 .

\begin{tabular}{|c|c|c|}
\hline Divisão & Família & Gênero ou espécie \\
\hline \multirow[t]{4}{*}{ Chlorophyta } & Cladophoraceae & Chaetomorpha Kützing \\
\hline & & Cladophora Kützing \\
\hline & & Rhizoclonium Kützing. \\
\hline & Bryopsidaceae & Bryopsis J. V. Lamouroux spp. \\
\hline \multirow[t]{8}{*}{ Phaeophyta } & Ectocarpaceae & Acinetospora crinita (Charm. ex Harve in Hook) Kornmann \\
\hline & & Bachelotia antillarum(Grunov) Gerloff \\
\hline & & Ectocarpus Lyngby \\
\hline & & Feldmannia Hamel \\
\hline & & Hincksia J. E Gray \\
\hline & Sphacelariaceae & Sphacelaria brachygonia Montagne \\
\hline & & S. rigidula Kützing \\
\hline & & S. tribuloides Meneghini \\
\hline \multirow[t]{7}{*}{ Rhodophyta } & Ceramiaceae & $\begin{array}{l}\text { Centroceras clavulatum (C. Agardh in Kunth) Montagne in } \\
\text { Durieu de Maisonneuve }\end{array}$ \\
\hline & & Ceramium Roth spp. \\
\hline & Dasyaceae & Dasya C. Agardh spp. \\
\hline & & Heterosiphonia Montagne spp. \\
\hline & Rhodomelaceae & Herposiphonia Nägeli spp. \\
\hline & & $\begin{array}{l}\text { Ophidocladus simpliciusculus (P.Crouan \& H.Crouan) Falkenberg } \\
\text { Polysiphonia Greville spp. }\end{array}$ \\
\hline & & Pterosiphonia pennata (C. Agardh) Falkenberg \\
\hline
\end{tabular}

Tabela 2. Táxons mais representativos de algas coletados nos quadrados amostrados nas praias Rasa, Grande e Vermelha.

\begin{tabular}{|c|c|c|c|c|c|}
\hline Divisão & Família & Táxon & Rasa & $\begin{array}{r}\text { Praia } \\
\text { Grande }\end{array}$ & Vermelha \\
\hline \multirow[t]{5}{*}{ Chlorophyta } & Codiaceae & Codium decorticatum (Woodward) M. Howe & & ${ }^{*}$ & ${ }^{*}$ \\
\hline & Ulvaceae & Enteromorpha Link in Ness spp. & * & * & * \\
\hline & & Ulva fasciata Delile spp. & * & & \\
\hline & & U. lactuca Linnaeus spp. & * & * & * \\
\hline & & U. rigida C. Agardh) spp. & * & * & * \\
\hline \multirow[t]{10}{*}{ Phaeophyta } & Chordariaceae & Levringia brasiliensis (Montagne) A. B. Joly & * & & \\
\hline & Dictyotaceae & Dictyopteris delicatula Lamouroux & * & * & * \\
\hline & & Dictyota cervicornis Kützing & * & * & * \\
\hline & & Padina gymnospora (Kützing) Sonder & * & * & * \\
\hline & & Stypopodium zonale (Lamouroux) Papenfuss & & * & * \\
\hline & Sargassaceae & Sargassum C. Agardh spp. & * & * & * \\
\hline & & S. cymosum C. Agardh var. cymosum & & * & * \\
\hline & & S. filipendula C. Agardh var. filipendula & & * & \\
\hline & & S. vulgare C. Agardh var. vulgare & * & & \\
\hline & Scytosiphonaceae & Colpomenia sinuosa (Roth) Derbès \& Solier & * & & \\
\hline \multirow[t]{12}{*}{ Rhodophyta } & Ceramiaceae & Spyridia filamentosa (Wulfen) Harvey in Hook. & * & * & * \\
\hline & & S. hypnoides (Bory in Belanger) Papenfuss & * & * & * \\
\hline & Gelidiaceae & Gelidium J. V. Lamouroux spp. & & * & * \\
\hline & Gigartinaceae & $\begin{array}{l}\text { Chondracanthus acicularis (Roth) Fredericq } \\
\text { C. teedii (Mertens ex Roth) Fredericg }\end{array}$ & & * & \\
\hline & Gracilariaceae & Gracilaria cervicornis (Turner) J. Agardh & * & * & * \\
\hline & Halymeniaceae & Grateloupia C. Agardh spp. & & * & * \\
\hline & Hypneaceae & Hypnea spinella (C. Agardh) Kützing, & * & * & * \\
\hline & Phyllophoraceae & Gymnogongrus griffithsiae (Turner) Martius & & * & * \\
\hline & Rhodomelaceae & $\begin{array}{l}\text { Acanthophora spicifera (Vahl) Börgesen. } \\
\text { Chondria polyrhiza Collins \& Hervey }\end{array}$ & * & * & \\
\hline & & Laurencia J. V. Lamouroux spp. & * & & * \\
\hline & Rhodymeniaceae & Gelidiopsis planicaulis (W. R. Taylor) & & * & * \\
\hline & & $\begin{array}{l}\text { W. R. Taylor } \\
\text { Rhodymenia pseudopalmata (Lamouroux) Silva }\end{array}$ & & & * \\
\hline
\end{tabular}


A variação espaço-temporal nas três populações de $H$. musciformis foi obtida através da análise de variância (ANOVA), utilizando o teste de Tukey para detectar diferenças entre as médias. Estas análises foram escolhidas por serem consideradas robustas para desvios na normalidade (Underwood 1981; Zar 1996). A homogeneidade
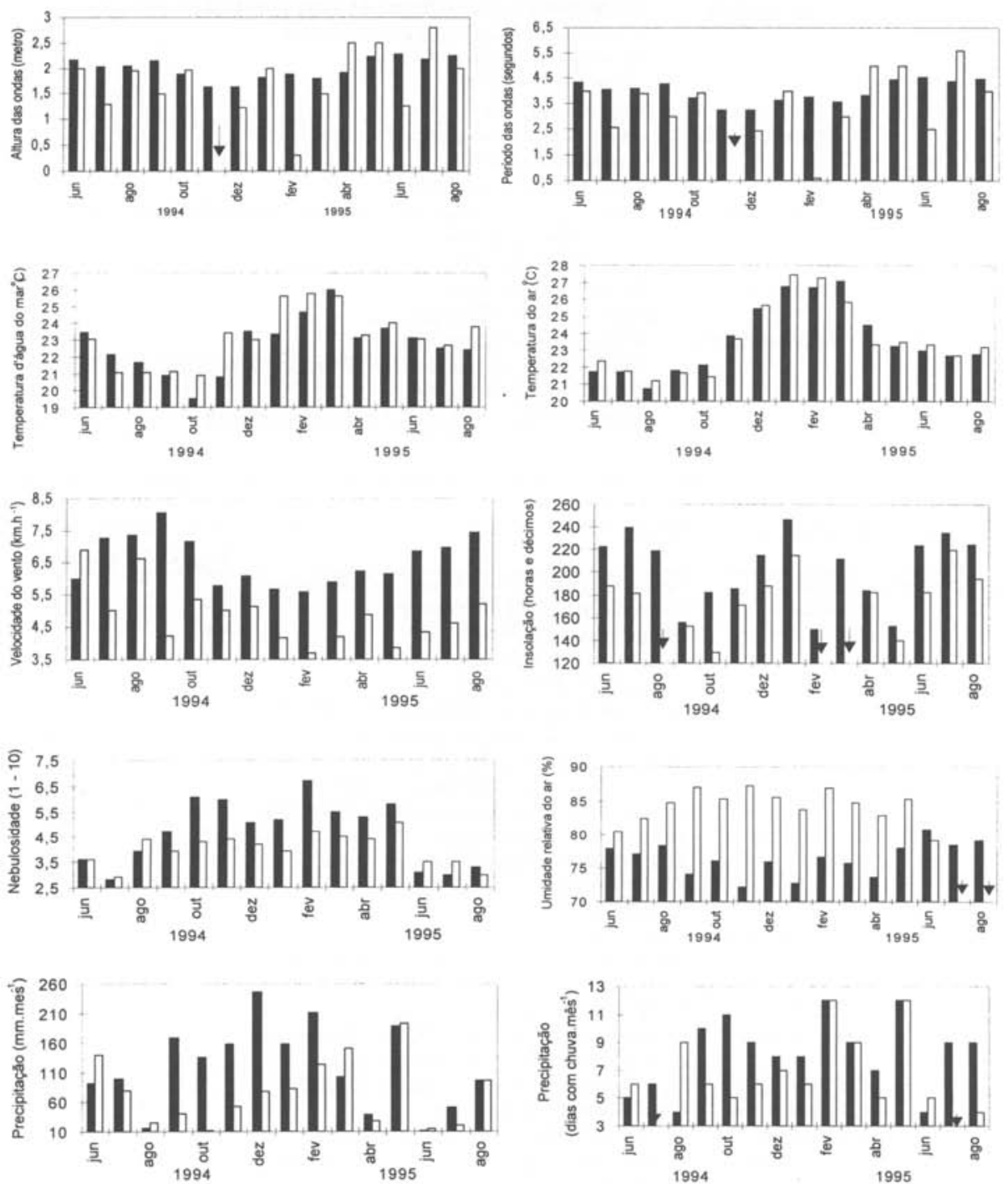

Figura 2. Dados meteorológicos da altura e período das ondas, temperaturas do ar e de água do mar e velocidade do vento, cedidos pela DHN, e dados da insolação, nebulosidade, umidade relativa do ar e precipitação, segundo o INMET. Setas indicam ausência de dados. Barras escuras indicam valores da região de Búzios e barras claras, à região de Sepetiba. 
das variâncias foi verificada através do teste de Cochran (Winner 1971) e, quando necessário, os dados foram transformados para raiz quadrada ou logaritmo (Zar 1996).

As interações entre os dados ambientais e as biomassas foram detectadas através do coeficiente de correlação de Pearson (Zar 1996). Para este cálculo foi utilizado o mês anterior ao da coleta bimestral da biomassa, que se assemelha ao dado referente ao de uma semana anterior à coleta. Utilizou-se valores referentes ao primeiro ano de coleta.

Os valores das biomassas foram apresentados como média \pm desvio padrão e o grau de confiança para todos os testes de significância foi estipulado em $95 \%$ (p e $\alpha=0,05$ ).

\section{Resultados}

Distribuição das macroalgas acompanhantes de H. musciformis

Praia Rasa - A comunidade algácea, coletada na área de amostragem, é formada por algas fortemente emaranhadas em forma de tapete com ca. $5 \mathrm{~cm}$ alt. Dentre as Chlorophyta, as biomassas de Ulvaceae (Enteromorpha spp., Ulva fasciata, U. lactuca e $U$. rigida) foram as que ocorreram em maiores proporções. Entre as Phaeophyta dominaram Padina gymnospora, Colpomenia sinuosa, Levringia brasiliensis, Sargassum vulgare var. vulgare e entre as Rhodophyta, ocorreram, principalmente, espécies de Laurencia spp., Hypnea spinella, Gracilaria cervicornis, Spyridia filamentosa, S. hypnoides e Acanthophora spicifera.

Em geral, os maiores percentuais foram de H. musciformis, seguido do grupo "filamentosa", Sargassum spp., Laurencia spp., Ulvaceae, Dictyotaceae (Dictyopteris delicatula e Dictyota cervicornis e H. spinella. Entre agosto/1994 e agosto/1995, os táxons mais representativos foram $\mathrm{H}$. musciformis, com $41 \%$ da biomassa, seguido do grupo "filamentosa", com $26 \%$ de representatividade e com percentuais abaixo de $10 \%$ ocorreram principalmente espécies de Sargassum, Laurencia e Ulva, Dictyotaceae e H. spinella. A partir outubro/1995 aumentou o percentual de Sargassum, que dividiu o substrato com H. musciformis e com o grupo "filamentosa" (Fig. 3).

Em cada coleta, a distribuição dos táxons no costão seguiu padrão parecido ao descrito acima, ou seja, as biomassas de $H$. musciformis e do grupo "filamentosa" revestindo o substrato em pelo menos cerca de $50 \%$, com exceção em dezembro/1994 e agosto/1996, quando aumentou o percentual de Ulvaceae, e a partir de fevereiro/1996, com o aumento de Sargassum spp. nos meses de fevereiro, junho e agosto (Fig. 4).

Praia Grande - Entre junho/1994 e junho/1995, predominaram os extensos bancos de Sargassum spp., com 68\% de biomassa, constituídos, principalmente, por S. cymosum var. cymosum e S. filipendula var. filipendula, representados por indivíduos com até $50 \mathrm{~cm}$ alt., tendo $H$. musciformis como epífita, que participou com $18 \%$ de biomassa. Abaixo ou igual a 6\%, ocorreram Codium decorticatum, o qual, no verão, mediu ca. $70 \mathrm{~cm}$ compr., Padina gymnospora e Gracilaria cervicornis, com plantas atingindo até $20 \mathrm{~cm}$ alt. Gelidium spp. e Gelidopsis planicaulis revestiram o substrato, abaixo da copa de Sargassum spp. (Fig. 5A, Tab. 2).

Entre abril a junho/1995, ocorreu queda na biomassa de todas as algas (ANOVA, $\mathrm{F}=8,70, \mathrm{p}=0,01)$, a qual passou de $8,58 \pm 4,38 \mathrm{~g} \cdot \mathrm{m}^{-2}$ para $2,85 \pm 2,69 \mathrm{~g} \cdot \mathrm{m}^{-2}$. No período seguinte, entre junho e agosto, houve nova diminuição da biomassa (ANOVA, F=5,12, 
A

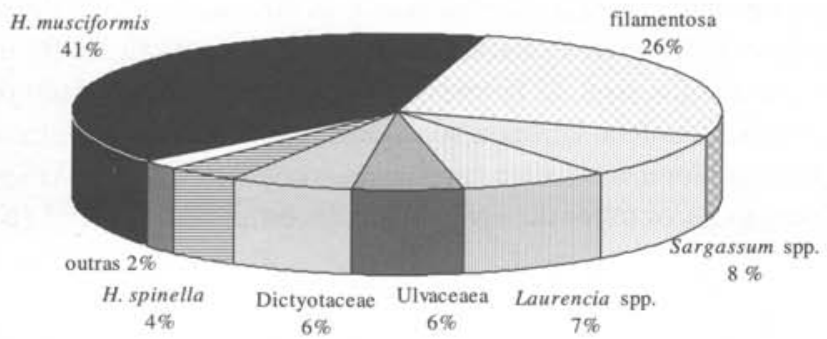

filamentosa $23 \%$

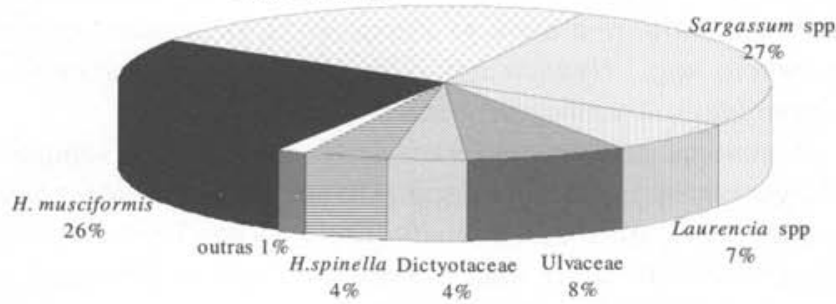

B

Figura 3. Táxons de algas coletados na praia Rasa. A: de agosto/1994 a agosto/1995 e B: de outubro/1995 a agosto/1996.

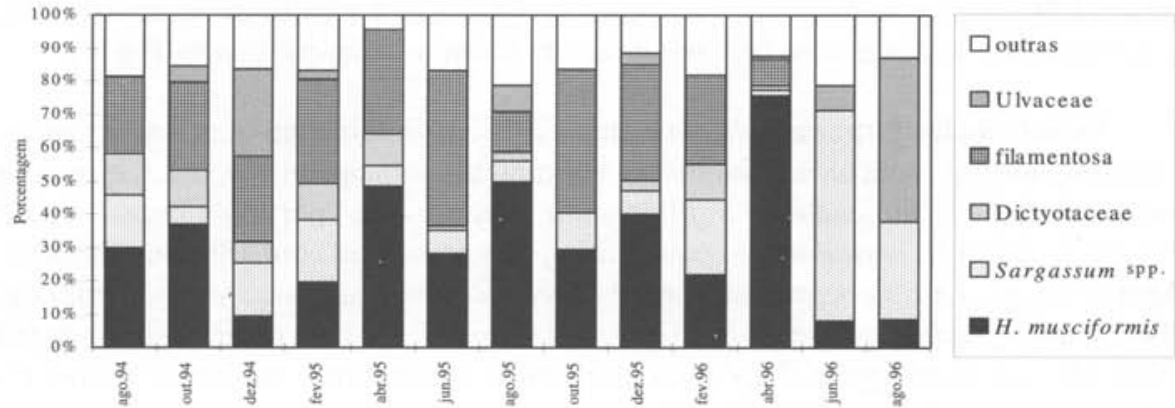

Figura 4. Táxons de algas predominantes na praia Rasa, em cada coleta, de agosto/1994 a agosto/1996. 


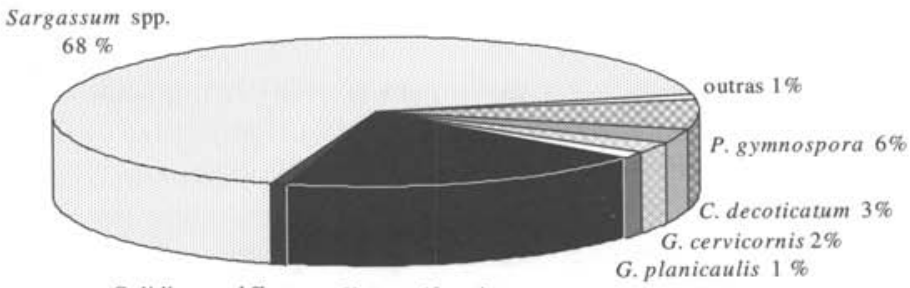

A

Gelidiaceae $1 \%$

H. musciformis

$18 \%$

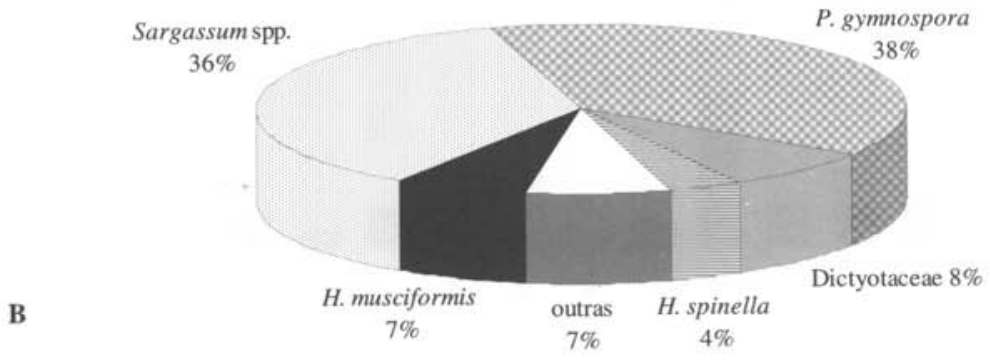

Figura 5. Táxons de algas coletados na praia Grande A: taxons coletados de junho/1994 a junho/1995 e B: de agosto/1995 a junho/1996.

$\mathrm{p}=0,04)$, passando para $0,48 \pm 0,58 \mathrm{~g} \cdot \mathrm{m}^{-2}$. Essa diminuição na biomassa da praia Grande acarretou a transformação da fisionomia da comunidade algácea. Em vez de indivíduos de grande porte de Sargassum observou-se várias populações algáceas com poucos centímetros de altura (tufos em forma de tapete), predominando espécies de Chlorophyta e Rhodophyta.

Na Fig. 5B observa-se que, a partir de agosto, época da drástica queda da biomassa, predominaram Padina gymnospora e Sargassum spp., compondo o total de $74 \%$ de biomassa, enquanto que os demais táxons tiveram biomassa reduzida a percentuais iguais ou abaixo de $8 \%$. Entre estes, em geral, o substrato vivo Sargassum spp. teve como epífitas as Dictyotaceae (D. delicatula e D. cervicornis), H. spinella e $H$. musciformis. Foram agrupadas em "outras", cobrindo o substrato em $7 \%$, as Ulvaceae (Enteromorpha spp., U. lactuca e U. rigida), Stypopodium zonale, Chondracanthus acicularis, C. teedii, Gymnogongrus gryffithsiae, Spyridia filamentosa, S. hypnoides, Grateloupia spp., Chondria polyrhiza e algumas das algas agrupadas como "filamentosa".

A proporção destas algas variou com as estações do ano. Em geral, de junho/1994 a junho/1995, predominaram os extensos bancos de Sargassum spp., com H. musciformis como epífita, em menor proporção. Entretanto, em dezembro/1994, os bancos de $H$. 
musciformis, Codium decorticatum, Padina gymnospora e Sargassum spp. predominaram e apresentaram biomassas semelhantes entre si (ANOVA, $\mathrm{F}=1,35, \mathrm{p}=0,28)$. Em abril/ 1995, o percentual da biomassa de H. musciformis igualou-se ao de Sargassum spp. (ANOVA, $\mathrm{F}=0,21, \mathrm{p}=0,65$ ). De junho para agosto/1995, quando ocorreu a redução na biomassa total de algas (ANOVA, $\mathrm{F}=5,12, \mathrm{p}=0,04$ ), o percentual de $P$. gymnospora passou a ser maior do que o de Sargassum spp., a partir de outubro (Fig. 6).

Praia Vermelha - A comunidade algácea apresentou fisionomia parecida à da comunidade da praia Grande, diferindo apenas quanto ao menor porte dos indivíduos de Sargassum spp. (ca. 30cm alt.). Entre junho/1994 e junho/1995 (Fig. 7A), apresentou

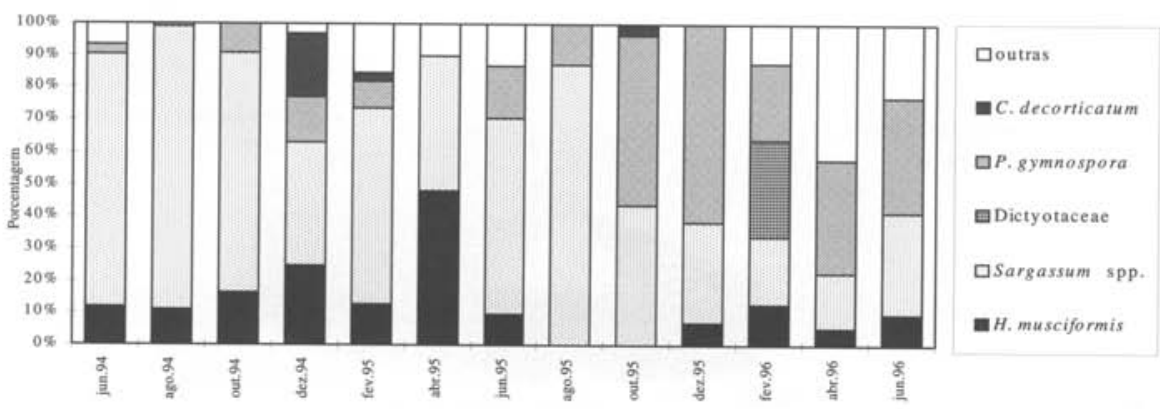

Figura 6. Táxons de algas predominantes na praia Grande, em cada coleta, entre junho/1994 e junho/1996.

alta percentagem de espécies de Sargassum, 49\%, principalmente representado por $S$. cymosum var. cymosum, portando como epífitas Dictyotaceae, $25 \%$ (D. cervicornis e D. delicatula), H. musciformis, $10 \%$, e H. spinella, $3 \%$.

Recobrindo o costão rochoso em 5\% ocorreram Ulvaceae (Enteromorpha spp., U. lactuca e U. rigida), Codium decorticatum, Stypopodium zonale, Rhodymenia pseudopalmata, Gracilaria cervicornis, Gymnogongrus griffithsiae, além de espécies de Gelidium, Chondracanthus, Grateloupia e Spyridia. Estes táxons foram incluídos em "outras". Em percentuais de 3\% ocorreram Gelidiopsis planicaulis e Padina gymnospora e, de $2 \%$, as espécies do grupo "filamentosa" .

De junho para agosto/1995 ocorreu drástica queda na biomassa de todas as algas da praia Vermelha (ANOVA, $\mathrm{F}=16,23, \mathrm{p}=0,002$ ), que passou de $120,13 \pm 66,08 \mathrm{~g} \cdot \mathrm{m}^{-2}$ para $17,60 \pm 12,64 \mathrm{~g} \cdot \mathrm{m}^{-2}$. Como conseqüência, a vegetação apresentou biomassa escassa, com exemplares medindo poucos centímetros de altura, predominando G. planicaulis, $35 \%$, H. musciformis, $31 \%$, o grupo "filamentosa", $18 \%$, Sargassum spp., $12 \%$, e Gelidium spp., 4\% (Fig. 7B).

A Fig. 8 mostra a variação no percentual da biomassa, ao longo das estações do ano, na praia Vermelha. No inverno/1994, a percentagem de $H$. musciformis foi de no máximo 2\%, enquanto a de Sargassum spp. esteve próximo a 77\%, e a de Dictyotaceae (D. delicatula e D. cervicornis), entre 6 e $11 \%$. Na primavera aumentaram os percentuais de H. musciformis e os de Dictyotaceae, enquanto no verão, houve aumento nos percentuais de Padina gymnospora (22 \%) e "filamentosa" (8\%). A partir do outono/1995 (abril), 


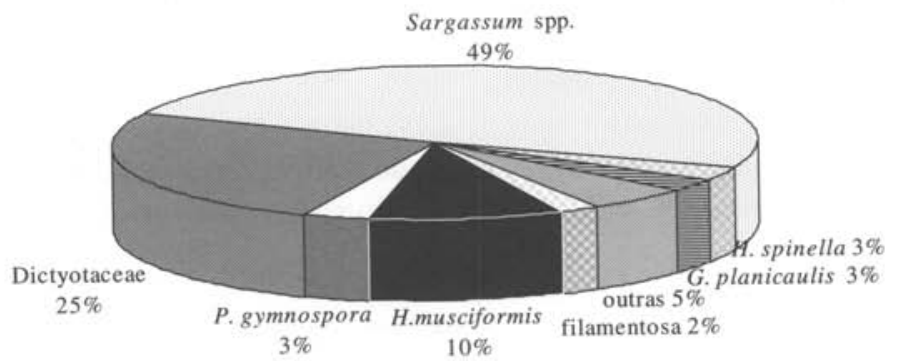

B

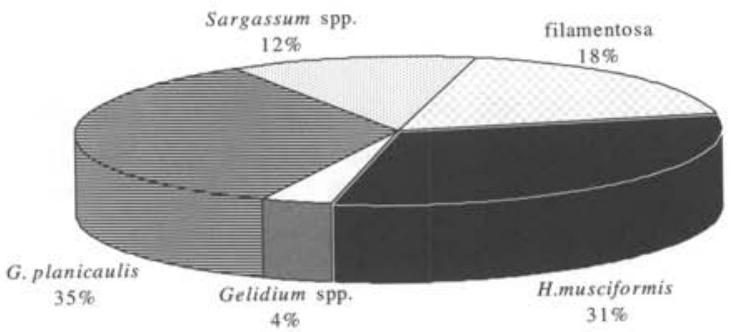

Figura 7. Táxons de algas coletados na praia Vermelha. A: de junho/1994 a junho/1995 e B: de agosto/1995 a junho/1996.

período da queda de biomassa, a fisionomia da comunidade de algas era formada de tufos compostos, principalmente por Gelidiopsis planicaulis, que alcançou o percentual de biomassa de $83 \%$ em fevereiro/1996 e depois, por espécies do grupo "filamentosa" e por espécies de Gelidium, Chondracanthus, Grateloupia, Spyridia, Hypnea e Laurencia.

Distribuição espaço-temporal de H. musciformis.

Praia Rasa - A biomassa de H. musciformis diferiu significativamente entre agosto/ 1994 e agosto/1996 (Fig. 9 e Tab. 3), variando de 8,06 $\pm 6,78$ a 58,84 $\pm 25,20 \mathrm{~g} . \mathrm{m}^{-2}$. Entre

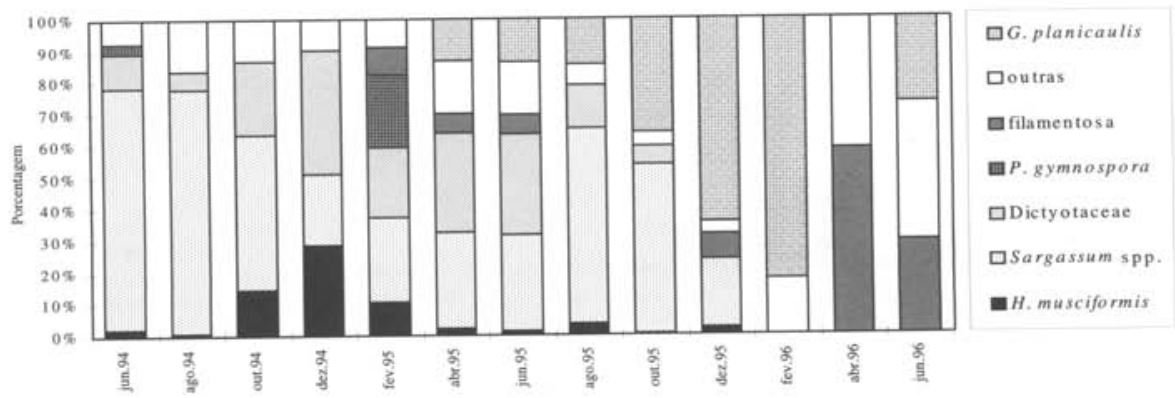

Figura 8. Táxons de algas predominantes na praia Vermelha, em cada coleta, de junho/1994 a junho/1996. 
a primavera (outubro/1994) e o outono (abril/1995), constatou-se queda na mesma, quando ocorreu grande número de ápices esbranquiçados. Esta biomassa recuperou-se no outono (ANOVA, $\mathrm{F}=8,68, \mathrm{p}<0.001$ ).

Foram obtidas correlações significativas entre a biomassa de $H$. musciformis e alguns fatores ambientais, entre eles a umidade relativa do $\operatorname{ar}(r=0,77, n=7, a=0,03), o$ período das ondas $(r=0,69, n=7, a<0,05)$ e a altura das ondas $(r=0,78, n=7, a=0,03)$.
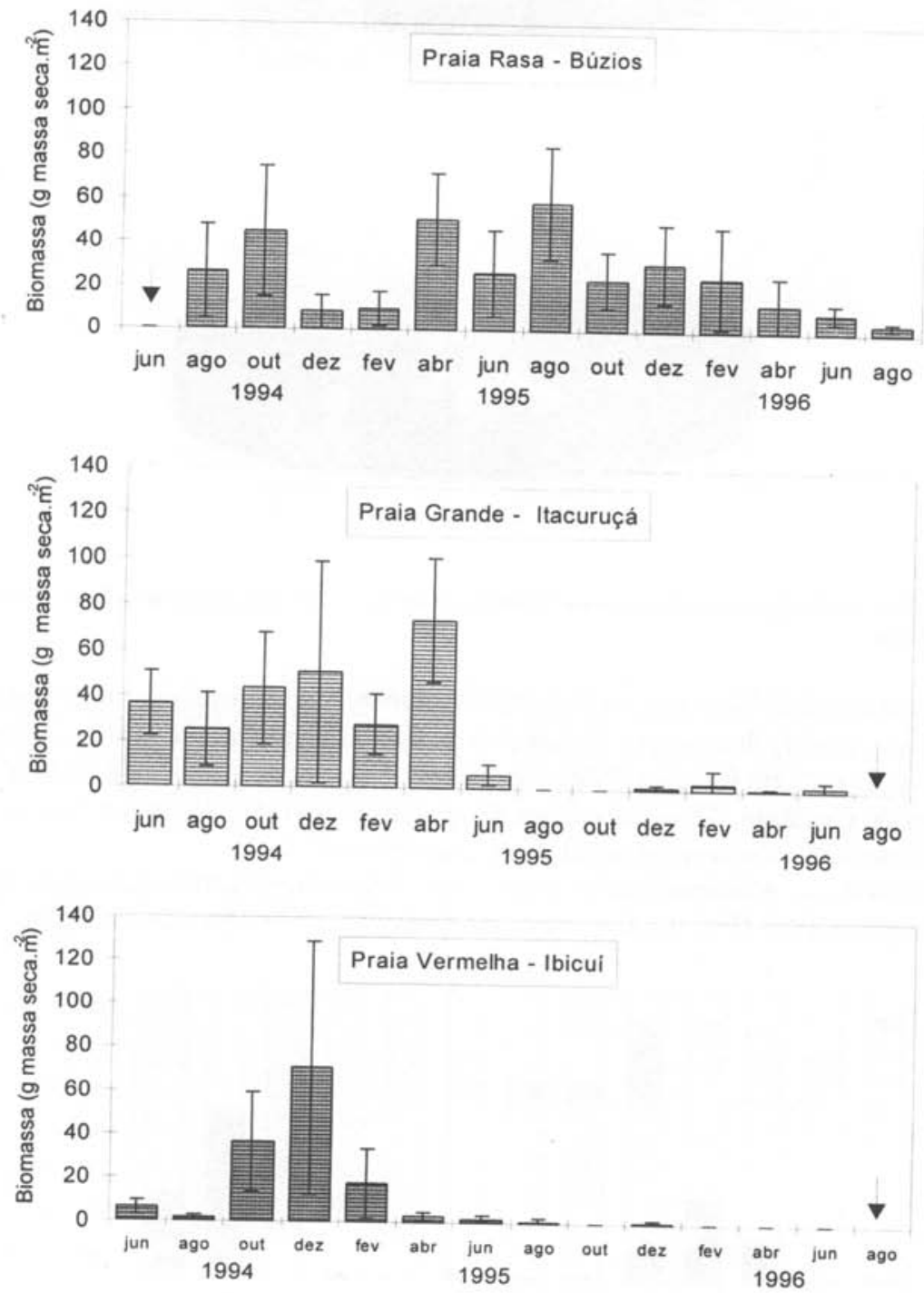

Figura 9. Biomassa de H. musciformis coletada na praia Rasa e nas praias Grande e Vermelha ao longo do tempo. Média \pm desvio padrão. Seta indica ausência de dados. 
Tabela 3. Análises de variância da biomassa ( $\mathrm{g}$ massa seca.m ${ }^{-2}$ ) de $H$. musciformis nas diferentes estações de coleta.

\begin{tabular}{lccccc}
\hline Estações de coleta & Período & Media \pm desvio padrão & g.l. & F & Valor p \\
\hline Pr. Rasa & 08/1994 a 08/1996 & $25,12 \pm 23,88$ & 97 & 6,41 & $<0,001$ \\
Pr. Grande & 06/1994 a 06/1995 & $37,36 \pm 30,86$ & 48 & $7,74^{(1)}$ & $<0,001$ \\
Pr.Vermelha & 06/1994 a 06/1995 & $20,40 \pm 33,31$ & 48 & $10,80^{(2)}$ & $<0,001$ \\
\hline
\end{tabular}

$\mathrm{F}=$ Razão das variâncias, g. $\mathrm{l}$. = graus de liberdade ${ }^{(1)}$ dados transformados para raiz quadrada $\mathrm{e}^{(2)}$ para logaritmo.

Praia Grande - No período de junho/1994 até junho/1995, a biomassa epifítica de H. musciformis diferiu ao longo do tempo (Fig.9, Tab. 3). Nesse período, houve aumento significativo na biomassa, entre fevereiro e abril/1995 (ANOVA, $F=16,21, p=0,002$ ), chegando a $72,51 \pm 27,00 \mathrm{~g} \cdot \mathrm{m}^{-2}$. Queda marcante (ANOVA, $\mathrm{F}=55,72, \mathrm{p}<0,001$, dados transformados para logaritmo) foi constatada de abril para junho/1995. A partir desta data, a biomassa de $H$. musciformis e das algas acompanhantes mantiveram-se reduzidas, variando de 0 a $6,15 \pm 4,23 \mathrm{~g} \cdot \mathrm{m}^{-2}$, além da ausência de $H$. musciformis entre agosto e outubro/1995. A biomassa de H. musciformis da praia Grande correlacionou-se positivamente com a nebulosidade $(r=0,89, \mathrm{n}=7, \mathrm{a}=0,005)$, quando analisados os fatores ambientais coletados entre junho/1994 a junho/1995, período de altos valores de médias de biomassa de $H$. musciformis.

Praia Vermelha - A biomassa de H. musciformis variou de $70,60 \pm 58,20 \mathrm{~g} \cdot \mathrm{m}^{-2} \mathrm{a}$ $1,49 \pm 1,29 \mathrm{~g} . \mathrm{m}^{-2}$, diferindo significativamente no período de junho/1994 a junho/1995 (Fig. 9, Tab. 3). Após este período, a biomassa de H. musciformis reduziu-se ainda mais, em média com $0,3 \pm 0,7 \mathrm{~g} \cdot \mathrm{m}^{-2}$. Comparando o período de agosto a outubro/1994, verificou-se aumento da biomassa do inverno para a primavera (ANOVA, $\mathrm{F}=36,45$, $\mathrm{p}<0,001$, dados transformados para logaritmo). Entretanto, drástica diminuição na biomassa de $H$. musciformis ocorreu de fevereiro para abril/1995 (ANOVA, $\mathrm{F}=7,34$, $\mathrm{p}=0,02$ ), não havendo mais recuperação da biomassa dessa alga.

De acordo com a Tab. 4, comparando as biomassas das populações sublitorâneas epifíticas de H. musciformis da baía de Sepetiba (praias Grande e Vermelha) em cada coleta, a biomassa de $H$. musciformis da praia Grande apresentou valor superior em junho e agosto/1994 e abril/1995.

Tabela 4. Análises de variância para comparar as biomassas (g massa seca.m² ${ }^{-2}$ de H. musciformis, em cada coleta, na Praia Grande (PG) e praia Vermelha (PV).

\begin{tabular}{lcclc}
\hline Mês & g.l. & $\mathrm{F}$ & Valor P & Teste de Tukey \\
\hline $06 / 1994$ & 13 & 16,26 & $=0,002$ & PG $>$ PV \\
$08 / 1994$ & 13 & $17,9^{(1)}$ & $<0,001$ & PG $>$ PV \\
$10 / 1994$ & 13 & 0,32 & $=0,58$ & - \\
$12 / 1994$ & 13 & 0,61 & $=0,45$ & - \\
$02 / 1995$ & 13 & 0,96 & $=0,35$ & - \\
$04 / 1995$ & 13 & 47,67 & $<0,001$ & PG $>$ PV \\
$06 / 1995$ & 13 & $6,00^{(1)}$ & $=0,31$ & - \\
\hline
\end{tabular}

$\mathrm{F}=$ Razão das variâncias, g. 1. = graus de liberdade ${ }^{1)}$ dados transformados para logaritmo. 


\section{Discussão}

Os resultados obtidos confirmaram a existência da sinergia entre os fatores ambientais (Murthy et al. 1989,;Faccini 1998). As biomassas de H. musciformis variaram nas três áreas (ANOVA, p <0,001), não havendo padrão sazonal comum aos três locais. Resultados semelhantes foram obtidos em populações no Estado de São Paulo (Faccini 1998). Observou-se que um mesmo fator ambiental causou interferência diferenciada no crescimento desta alga de acordo com o local de coleta. Levando em consideração o substrato, na praia Rasa, as algas formavam densas almofadas emaranhadas fixas ao substrato, que é uma maneira evitar a forte dessecação por emersão e proteger-se das fortes ondulações (Kain \& Norton 1990).

Durante o período estudado, a população litorânea de Sargassum spp. não serviu como hospedeiro disponível para $\mathrm{H}$. musciformis, enquanto nas praias Vermelha e Grande, os exemplares de Sargassum atuaram como importante substrato disponível para as populações sublitorâneas de $H$. musciformis. Portanto, nesses dois ambientes a relação hóspede e hospedeiro foi muito mais intensa. Na praia Grande, em dezembro/ 1994, a redução de Sargassum spp. deve ter interferido na flutuação da biomassa de $H$. musciformis, na qual o percentual da biomassa diminuiu de 25 para $13 \%$ em fevereiro/ 1995. Esta redução pode ter sido ocasionada pelo período de senescência de Sargassum, que já foi observado entre dezembro e janeiro (Paula 1978), e/ou pela competição por espaço entre Sargassum, Codium decorticatum e Padina gymnospora (Fig. 6), reduzindo assim este substrato vivo disponível para fixação de $\mathrm{H}$. musciformis. Ressalta-se que raras vezes se observou exemplares de $H$. musciformis sobre $P$. gymnospora e nunca foram vistos sobre $C$. decorticatum (observação pessoal); por outro lado, é comum essa carragenófita ocorrer na porção superior do talo de Sargassum spp. (Széchy \& Paula 1997).

Nas coletas bimestrais anteriores ao predomínio de Sargassum spp. na praia Rasa (anteriores às quatro últimas coletas), outras espécies acompanhantes, como as Dictyotaceae (Dictyopteris delicatula e Dictyota cervicornis), Laurencia spp. e o grupo "filamentosa", participaram da competição pelo substrato rochoso. Em ocasiões mais estressantes, como em dezembro/1994, ocorreu queda na biomassa de H. musciformis, aumentando substancialmente os percentuais das Ulvaceae (Enteromorpha spp., Ulva fasciata, U. lactuca e $U$. rigida), espécies que são mais resistentes a amplo gradiente de salinidade (Reis-Santos 1991). Em fevereiro do ano seguinte, a flora iniciou seu processo de restabelecimento, figurado pelo aumento das outras espécies e diminuição das Ulvaceae.

Apesar da semelhança entre a fisionomia das comunidades sublitorâneas das praias da baía de Sepetiba, ambas com predomínio de extensos bancos de Sargassum spp., constatou-se que na praia Vermelha, local com maior movimentação de água, as Dictyotaceae (D. delicatula e D. cervicornis) competiram por espaço com $H$. musciformis, enquanto que, na praia Grande, o percentual dessas epífitas foi menor. Consolidando este fato, ao comparar as biomassas de H. musciformis das duas praias, em abril/1995, a menor biomassa foi encontrada na praia Vermelha, o que deve ser consequuência da competição desta espécie com essas Dictyotaceae. Sendo assim, parece 
que essas feofíceas contribuíram para menor proporção de $H$. musciformis devido à competição por luz, nutrientes e espaço (Széchy \& Paula 1997; Kain \& Norton 1990). Schenkman (1980), Berchez (1990) e Faccini (1998) discutem amplamente a influência da disponibilidade de substrato e competição encontrados em seus cultivo com $H$. musciformis. Estes fatos evidenciam a existência de diferentes espécies competidoras em diferentes locais.

A movimentação de água do mar, que provoca a freqüência e a altura das ondas, interferiu de modo diferenciado no crescimento das algas nas distintas populações de H. musciformis estudadas. Na população litorânea, que cresce diretamente sobre as rochas (praia Rasa), a altura e a freqüência das ondas, aliadas a umidade relativa do ar, protegeu esta população contra a dessecação, favorecendo o crescimento desta carragenófita. Já no inverno de 1994, esse fator abiótico, movimentação de água do mar, ocasionou efeito contrário na população sublitorânea e epifítica da praia Vermelha (baía de Sepetiba), devido as fortes ondulações, a maior inclinação do substrato $\left(30^{\circ}\right) \mathrm{e}$ as baixamares diurnas que fragmentaram o talo de H. musciformis. É conhecido o efeito negativo da forte movimentação de água sobre Rhodophyta (Kain \& Norton 1990). Comparando as biomassas de $H$. musciformis das praias da baía de Sepetiba (Grande e Vermelha), no inverno, constatou-se menor biomassa na praia Vermelha, que possui movimentação de água maior que a da praia Grande (Reis 1998). Fortes tempestades causaram perda da biomassa de $\mathrm{H}$. musciformis no Estado de São Paulo (Schenkman 1980; 1989; Berchez et al. 1993) e da Bahia (Wallner et al. 1992), como também na Índia (Rao 1970).

Na praia Grande, considerada o local com menor movimentação de água dos três estudados (Reis 1998), a luz parece ter sido o principal fator responsável pela queda na biomassa de $H$. musciformis. A maior penetração dos raios solares como resposta à fraca movimentação da água (menor turbulência) deve ter ocasionado a tendência de diminuição na biomassa desta alga, em agosto/1994, constatada pela ocorrência de altos valores de insolação e baixos de nebulosidade e ondulações, nos meses anteriores (junho e julho/1994), além da correlação positiva entre a biomassa e a nebulosidade. Guist et al. (1982) constataram que a forte intensidade luminosa pode ser prejudicial ao crescimento de $H$. musciformis.

As respostas obtidas no presente estudo fortalecem a afirmativa da existência de variação no comportamento sazonal na biomassa de $H$. musciformis de diferentes locais (Durako \& Dawes 1980; Berchez \& Oliveira 1990; Faccini 1998). Provavelmente, os fatores que mais contribuem para o aumento na biomassa desta alga são os fatores ambientais em microescala, ou seja, os locais. Este fato foi observado quando comparadas as amostras das biomassas de H. musciformis de duas áreas, uma com $100 \mathrm{~m}$ e outra com $5 \mathrm{~m}$. A área de $100 \mathrm{~m}$ apresentou maior variabilidade da biomassa entre as amostras, que foi atribuída à maior diversidade de microambientes neste local (Faccini 1998). Nesse contexto, outros exemplos podem ser anexados a estas respostas, como aqueles obtidos no Havaí e na Índia, onde as maiores biomassas de $H$. musciformis foram encontradas no inverno (Rao 1970; Mshigeni 1977; Murthy et al. 1989) e as menores, na primavera até meados do verão (Mshigeni 1977; Murthy et al. 1989). Na Flórida, EUA, o período de maiores biomassas foi antecipado para o outono, 
prolongando-se até o inverno, e o período de menores biomassas, foi no verão (Durako \& Dawes 1980). Esse baixo valor na biomassa (no verão) foi encontrado na praia Rasa e nos Estados da Bahia (Wallner et al. 1992) e de São Paulo (Berchez et al. 1993). Ocorreram, ainda, decréscimos na biomassa de $H$. musciformis durante o inverno, nas populações da praia Vermelha, do Estado de São Paulo (Schenkman 1980;1989) e da Bahia (Wallner et al. 1992). Contrariando os resultados encontrados na praia Vermelha, outro decréscimo da biomassa desta alga foi observado na primavera, em uma população desta alga em São Paulo (Schenkman 1980; 1989).

A população litorânea e epilítica de $H$. musciformis na praia Rasa sofreu mais as fortes condições ambientais encontradas no verão do que as populações sublitorâneas da baía de Sepetiba, inclusive a ação dos fatores ambientais deve ter sido exacerbada no costão, com a pouca inclinação deste local. O decréscimo na biomassa desta alga, de dezembro/1994 a fevereiro/1995, deve ter sido induzido pelos altos valores da temperatura do ar e da água, esta última conhecida como um dos fatores que controla a sazonalidade das espécies litorâneas (Kain \& Norton 1990). Além da forte insolação e da baixa salinidade, a qual deve ter sido ocasionada pela alta precipitação, acima de $150 \mathrm{~mm}$. $\mathrm{mes}^{-1}$, foi observado que as baixas salinidades podem reduzir a taxa fotossintética do organismo (Kain \& Norton 1990). Como conseqüência, foram observados ápices esbranquiçados, que indicam talos danificados (Schenkman 1986; Berchez et al. 1993). Berchez et al. (1993) e Faccini (1998) não conseguiram relacionar a interferência da temperatura no crescimento da biomassa das populações estudadas no Estado de São Paulo, o que pode ser explicado pelo grau de exposição de $H$. musciformis ao ar em costão, que deve ter inclinação bem menos acentuada que aquele da praia Rasa.

A partir do inverno de 1995 , além dos fatores abióticos interferindo na biomassa algácea nas praias da baía de Sepetiba, houve interferência antrópica causada pelo início das obras de dragagens para ampliação do Porto de Sepetiba, iniciadas em meados de abril/1995 (contato verbal com engenheiro da Gerência de Dragagem da Companhia Docas do Rio de Janeiro - GEDRAG/CDRJ). Conseqüentemente, devem ter aumentado a turbidez de água e acarretado a remobilização de elementos químicos, que por sua vez, devem ter modificando a qualidade da massa de água. Nesse período foram observados aumentos nos índices de concentração de metais pesados nos talos de Padina gymnospora (G. Amado Filho, comunicação pessoal), além das respostas obtidas em experimentos in vitro, demonstrando que a adição de pequenas concentrações de zinco, como $20 \mathrm{mg} . \mathrm{L}^{-1}$, podem inibir o crescimento de $H$. musciformis em apenas uma semana de cultivo (Amado Filho 1996; Amado Filho et al. 1997). Nessa ocasião, diminuíram os percentuais desta espécie nas estações de coleta da baía de Sepetiba e predominaram espécies de feofíceas, como Padina e Sargassum, que possuem a habilidade de tolerar e acumular metais pesados (Amado Filho et al. 1997).

A imprensa divulgou a grande mortandade de pescado nessa baía (Gonçalves $e t$ al. 1996). Em fevereiro/1996, ocorreu o transbordamento de metais pesados da Companhia Ingá Mercantil, fábrica de zinco em Itaguaí, após forte temporal (Anderson \& Gonçalves 1996; Gonçalves \& Intrator 1996; Javoski 1996), que foi acompanhado pelo decréscimo total da comunidade algácea. Esses fatos sugerem a influência negativa da ação antrópica no crescimento da biomassa algácea nesta baía. 
A alta taxa de crescimento de $H$. musciformis observada em outros estudos, acima de $10 \%$. dia ${ }^{-1}$ (Berchez \& Oliveira 1990; Schenkman 1989; Russel \& Balazans 1994; Faccini 1998), proporciona alto poder de recuperação da biomassa conforme constatado no estudo sobre a recuperação desta alga em populações no Estado de São Paulo, com média de recuperação de $87 \%$ mês $^{-1}$ (Faccini 1998). As populações do Estado do Rio de Janeiro podem ser boas candidatas a exploração se for considerado que provavelmente possuem o mesmo poder de recuperação, visto o aumento da biomassa após períodos desfavoráveis de crescimento. Entretanto, deve-se, também considerar a ocorrência de decréscimos na biomassa como conseqüência de ação antrópica.

De acordo com os resultados obtidos, conclui-se que, para viabilizar a exploração racional de $H$. musciformis, assim como para proteger os ecossistemas marinhos, tornase necessário o estudo da comunidade no local a ser explorado, uma vez que a biomassa dessa espécie, em geral, é inconstante no tempo e no espaço (Berchez \& Oliveira 1990; Faccini 1998).

\section{Agradecimentos}

Ao Conselho Nacional de Desenvolvimento Científico e Tecnológico - CNPq, através de bolsa para a segunda Autora; à Coordenação de Aperfeiçoamento de Pessoal de Nível Superior - CAPES, pelo apoio financeiro, ao Instituto Nacional de Meteorologia/Distrito Sexto de Meteorologia/Ministério da Agricultura e do Abastecimento (INMET) e ao Banco de Dados Oceanográficos da Diretoria de Hidrografia e Navegação/Ministério da Marinha (DHN), pelo fornecimento de dados meteorológicos; aos colegas do Jardim Botânico, pelo constante apoio e críticas.

\section{Referências bibliográficas}

Amado Filho, G. 1996. Metais pesados e macroalgas marinhas: resultados de campo, ensaios em laboratório e localização ultraestrutural. Tese de Doutorado. Universidade Federal do Rio de Janeiro, Rio de Janeiro.

Amado Filho, G.; Karez, C. S.; Andrade, L. R.; Yoneshigue-Valentin, Y. \& Pfeifer, W. C. 1997. Effects on growth and acummulation of zinc in six seaweeds species. Ecotoxicology and Environmental Safety 37: 223-228.

Anderson C. \& Gonçalves, L. 1996. Estado intervém em fábrica de zinco de Itaguaí. O Globo, Rio de Janeiro, 27 fev, Rio, p.18.

Barcellos, C. 1995 Geodinâmica de cádmio e zinco na Baia de Sepetiba. Tese de Doutorado. Universidade Federal Fluminense, Rio de Janeiro.

Berchez, F. A. S. 1990. Ensaios de maricultura da alga Hypnea musciformis (Rhodophyta, Gigartinales) no litoral do Estado de São Paulo. Tese de Doutorado. Universidade de São Paulo, São Paulo.

Berchez, F. A. S. \& Oliveira Filho, E. C. 1990. Maricultural essays with the carragenophyte Hypnea musciformis in São Paulo, Brasil. Pp. 89-94. In: E. C. Oliveira Filho, Kautsky, N. (Ed.), Cultivation of seaweeds in Latin America, Universidade de São Paulo, São Paulo.

Berchez, F. A. S.; Pereira, R. T. L. \& Kamiya, N. F. 1993. Culture of Hypnea musciformis (Rhodophyta - Gigartinales) on artificial substrates attached to linear ropes. Hydrobiologia 260/261: 415-420.

Câmara-Neto, C. 1987. Seaweed culture in Rio Grande do Norte, Brazil. Hidrobiologia 151/152: 363367.

Câmara-Neto, C.; Araujo, R. A. \& Pereira, M. V. 1980. Feasibility of seaweed culture in north-east areas of Brazil-2. Culture of Hypnea musciformis (Wulfen) Lamour. and Gracilaria verrucosa (Hudson) 
Papenfuss close to reefes of Buzios - RN, Brazil. Symposium Coastal Aquaculture, Cochin, India Abstr. 112

Chapman, A. R. O. 1985. Demography. Pp. 251-268. In M. M. Littler \& D. S. Littler (Ed.), Handbook of phycological methods: ecological field methods: macroalgae. Cambridge University Press, New York.

De Wreede, R. E. 1985. Destructive (harvest) sampling. Pp. 147-160. In M. M. Littler \& D. S. Littler (Ed.), Handbook of phycological methods: ecological field methods: macroalgae. Cambridge University Press, New York.

Durako, M. J. \& Dawes, C. J. 1980. A comparative seasonal study of two populations of Hypnea musciformis from the east and west coasts of Florida, USA I. Growth and chemistry. Marine Biology 59: 151-156.

Faccini, A. L. 1988. Ecologia e manejo de um banco natural de Hypnea musciformis (Rhodophyta, Gigartinales) no litoral norte do estado de São Paulo. Dissertação de Mestrado. Universidade de São Paulo, São Paulo.

Friedlander, M. \& Lipkin, Y. 1982. Rearing of agarophytes and carragenophytes under field conditions in Eastern Mediterranean. Botanica Marina 25: 101-105.

Friedlander, M. \& Zelikovitch, N. 1984. Growth rates, phycocolloid yield and quality of the red seaweeds Gracilaria sp., Pterocladia capillacea, Hypnea musciformis and Hypnea cornuta in field studies in Israel. Aquaculture 40: 57-66.

Gonçalves, L.; Alves, M. E. \& Intrator, S. 1996. Feema avaliará vazamento na Baía de Sepetiba. O Globo, Rio de Janeiro, 25 fev 1996. Rio, p.38.

Gonçalves, L. \& Intrator, S. 1996. Fábrica poluidora em Itaguaí deverá ser interditada. O Globo, Rio de Janeiro, 26 fev Rio, p.9.

Guist, G. G.; Dawes, C. J. \& Castle, J. R. 1982. Mariculture of the red seaweed Hypnea musciformis. Aquaculture 28: $375-384$.

Haines, K. C. 1975. Growth of the carrageenan-producing tropical red seaweed Hypnea musciformis in surface water, $870 \mathrm{~m}$ deep water, effluent from a clam mariculture system, and in deep water enriched with artificial fertilizer or domestic sewage Pp. 1: 207-220. In: Xth Europen Symposium of Marine Biology.

Humm, H. J. \& Kreuzer, J. 1975. On the growth rate of the red alga Hypnea musciformis, in the Caribbean sea. Caribbean Journal of Science 15: 1-4.

Javoski, V. 1996. Mergulhador relata devastação na Baía de Sepetiba, O Globo, Rio de Janeiro, 28 fev. Rio, p.17.

Kain, J. M. \& Norton, T. A. 1990. Marine Ecology. Pp. 377-422. In: K.M. Cole \& R. G. Sheath (Ed.), Biology of the red algae. Cambridge Univ. Press, New York.

Lima, A. M.; Câmara-Neto, C.; Oliveira-Filho, E. C \& Araujo, R. A. 1981. Cultivo experimental de Hypnea musciformis e Gracilaria sp. em áreas protegidas por antigas linhas de costa (recifes) no litoral do Rio Grande do Norte. SUDENE, Estudos de Pesca 9: 95-107

Langston, R. W.; Haines, K. C. \& Lyon, R. E. 1977. Ammonia-nitrogen production by the bivalve mollusc Tapes japonica and its recovery by the red seaweed Hypnea musciformis in a tropical marine system. Hegölander wiss. Meeresunters 30: 217-229.

Mshigeni, K. E. 1977. Seasonal changes in the standing crops of three Hypnea species (Rhodophyta: Gigartinales) in Hawai. Botanica Marina 20: 303-306.

Murthy, M. S.; Ramakrisna, T.; Rao, Y. N. \& Ghose, D. K. 1989. Ecological studies on some agarophytes from Veraval coast (India) I. Effects of aereal conditions on the biomass dynamics. Botanica Marina 32: $515-520$.

Oliveira Filho, E. C. 1977. Algas marinhas bentônicas do Brasil. Tese de Livre-Docencia. Universidade de São Paulo, São Paulo.

Oliveira Filho, E. C. \& Berchez, F. A. S. 1987. Ensayos sobre el cultivo del alga roja Hypnea musciformis (hodophyta - Gigartinales) in Sao Paulo, SP Pp. 339-409. In J. A . Verreth; M. Carrillo; S. Zanuys \& E. A. Huisman (Ed.), Proceedings of the Tall Trabajo sobre Acuicultura en America Latina, Insat. Foundation for Science.

Oliveira Filho, E. C.; Paula., E. J., Berchez, F. A. S., 1990, Essays on the cultivation of tropical red seaweeds in tanks. Pp. 79-87. In: E. C. Oliveira Filho \& Kautsky, N. Cultivation of seaweeds in Latin America, Universidade de São Paulo, São Paulo.

Oliveira Filho, E. C.; Oliveira, M. C.; Saito, R. M. \& Garofalo, G. M. C. 1992. Carragenanas algas polivalentes. Ciência Hoje 14(81): 73-77. 
Paula, E. J. 1978. Taxonomia, aspectos biológicos e ecológicos do gênero Sargassum (Phaeophyta Fucales) em condições de laboratório. Dissertação de Mestrado. Universidade de São Paulo, São Paulo.

Rao, R. K. 1970. Studies on growth cycle and phycocolloid content in Hypnea musciformis (Wulf.) Lamour. Botanica Marina 13: 163-165.

Reis, R. P. 1998. Variação espaço-temporal de carragenana em Hypnea musciformis (Wulfen) Lamouroux (Rhodophyta - Gigartinales) em bancos naturais do Estado do Rio de Janeiro, Brasil. Tese de Doutorado. Universidade Federal do Rio de Janeiro, Rio de Janeiro.

Reis-Santos, R. P. 1991. Flora algal da Lagoa de Araruama, RJ. Dissertação de Mestrado, Universidade Federal do Rio de Janeiro, Rio de Janeiro.

Russel, D. J. \& Balazs, G. H. 1994. Colonization by alien marine alga Hypnea musciformis (Wulfen) J.Ag. (Rhodophyta:Gigartinales) in Hawaiian Islands and its utilization by the green turtle, Chelonia mydas L. Aquatic Botany 47: 53-60

Schenkman, R. P. F. 1980. Biomassa, crescimento e ficocolóide de Hypnea musciformis (Rhodophyta) no litoral do Estado de São Paulo, Brasil. Dissertação de Mestrado. Universidade de São Paulo, São Paulo.

Schenkman, R. P. F. 1986. Cultura de Hypnea (Rodophyta) in vitro como subsídio para estudos morfológicos, reprodutivos e taxonômicos. Tese de Doutorado. Universidade de São Paulo, São Paulo.

Schenkman, R. P. F. 1989. Hypnea musciformis (Rhodophyta): ecological influence on growth. Journal of Phycology 25: 192-196.

Szechy, M. T. M., Paula, E. J. 1997. Macroalgas epífitas em Sargassum (Phaeophyta - Fucales) do litoral dos estados do Rio de Janeiro e São Paulo, Brasil. Leandra 12: 1-10.

Tsuda, R. T. \& Abbot, I. A. 1985. Collection, handling, preservation and logistics. Pp. 68-81, In: M.M. Littler \& D. S. Littler (Ed.), Handbook of phycological methods: ecological field methods: macroalgae. Cambridge Univ. Press, New York.

Ugadim, Y. 1985. Estudos taxonômicos de Gelidium e Pterocladia (Gelidiales - Rhodophyta) do Brasil. Tese de Livre-Docencia. Universidade de São Paulo, São Paulo.

Underwood, A. J. 1981. Techniques of analysis of variance in experimental marine biology and ecology. Oceanography and Marine Biology Annual Review 19: 513-605.

Wallner, M.; Lobo, S.; Boccanera, N. \& Mendes da Silva, E. 1992. Biomass, carrageenan yield and reproductive state of Hypnea musciformis (Rhodophyta : Gigartinales) under natural and experimental cultivated conditions. Aquaculture Fisheries Manage 23: 443-451.

Winner, B. J. 1971. Statistical principles in experimental design. $2^{\text {nd }}$ edition. McGraw-Hill, Tokyo.

Wynne, M. J. 1998. A checklist of benthic marine algae of the tropical and subtropical western Atlantic: first revision, Nova Hedwigia 116: 1-155.

Yokoya, S. N. 1989. Influência da temperatura e salinidade no crescimento de algumas espécies de agarófitas e carragenófitas - implicações práticas e biogeográficas. Dissertação de Mestrado. Universidade de São Paulo, São Paulo.

Yoneshigue, Y. 1985. Taxonomie et ecologie des algues marines dans la région de Cabo Frio (Rio de Janeiro, Brésil). Tese (Docteur d'État-Sciences), Université d'Aix, Marseille.

Zar, J. H. 1996. Biostatistical analysis. Prentice-Hall International Editions, New Jersey. 